\title{
STEREOSELECTIVITY EFFECT ON Tb(III)-Eu(III) ENERGY TRANSFER IN COMPLEXES WITH GLUTAMIC ACID AND ALANINE
}

\author{
J. LEgENDZIEWICZ, E. HUSKowsKA \\ Institute of Chemistry, University of Wrocław, F. Joliot-Curie 14, 50-383 Wrocław, \\ Poland \\ W. STRĘK, E. ŁUKOWIAK AND J. SZTUCKI \\ Institute of Low Temperature and Structure Research, Polish Academy of Sciences \\ Okólna 2, 50-950 Wrocław, Poland
}

\begin{abstract}
The effect of stereoselectivity on energy transfer between $\mathrm{Tb}(\mathrm{III})$ and $\mathrm{Eu}$ (III) in crystals with glutamic acid and alanine was investigated. It was found that the stereoselectivity significantly changes the energy transfer in these systems. The theoretical model for the selective dependent energy transfer processes in lanthanide systems is developed and the nature of processes occurring in both systems under investigation briefly discussed.
\end{abstract}

PACS numbers: 33.50.-j, 34.20.--b, 76.30.Kg

\section{Introduction}

The effect of stereoselectivity on energy transfer in lanthanide complexes in solutions was for the first time reported by Brittain [1]. He found that energy transfer rate was higher in racemic systems than in handed ones. A quite opposite behaviour was observed by Legendziewicz et al. [2] for the system of glutamic acid with $\mathrm{Eu}(\mathrm{III})$ and $\mathrm{Tb}(\mathrm{III})$ ions in crystals. The theory of stereoselectivity effect on energy transfer between lanthanide ions developed by Sztucki and Stręk [3] suggested that the energy transfer rate should be higher in handed systems than in racemic ones.

In this paper the energy transfer between $\mathrm{Tb}(\mathrm{III})$ and $\mathrm{Eu}$ (III) in crystallized complexes with racemic and L-handed alanine and glutamic acid is investigated. It was found that the stereoselectivity may increase or decrease the energy transfer yield. This effect depends on concentration and structure of crystals. It may be also linked with inherent chirality of ligands, as has been shown on a basis of new theoretical model developed by us in this paper. 


\section{Experimental results}

The monocrystals of $\mathrm{Eu}(\mathrm{III})$ and $\mathrm{Tb}(\mathrm{III})$ ions with alanine $\mathrm{Ln}_{2}(\mathrm{Ala})_{4}\left(\mathrm{H}_{2} \mathrm{O}\right)_{8}\left(\mathrm{ClO}_{4}\right)_{6}$ and glutamic acids $\mathrm{Ln}_{2}(\mathrm{Glu})_{2}\left(\mathrm{H}_{2} \mathrm{O}\right)_{9}\left(\mathrm{ClO}_{4}\right)_{4}$ were synthetized as it was described by us [2]. The luminescence decay times were measured using a $\mathrm{N}_{2}$ laser as an excitation source and recorded by boxcar integrator BCI290 (ZWG Berlin). The decay times of ${ }^{5} D_{4}$ level of $\mathrm{Tb}(\mathrm{III})$ ion in the systems of $(\mathrm{Eu}, \mathrm{Tb})$-alanine and glutamic acid crystals are presented in Table.

TABLE

Decay times of ${ }^{5} D_{4}$ level of $\mathrm{Tb}(\mathrm{III})$ in (Eu,Tb)-alanine and glutamic acid crystals.

\begin{tabular}{|c|c|c|c|c|c|}
\hline \multirow[t]{2}{*}{$\mathrm{Eu}_{x} \mathrm{~Tb}_{1-x}$} & \multicolumn{2}{|c|}{ Lifetime $[\mu \mathrm{s}]$} & \multirow[t]{2}{*}{$\mathrm{Eu}_{x} \mathrm{~Tb}_{1-x}$} & \multicolumn{2}{|c|}{ Lifetime $[\mu \mathrm{s}]$} \\
\hline & $T=300 \mathrm{~K}$ & $T=77 \mathrm{~K}$ & & $T=300 \mathrm{~K}$ & $T=77 \mathrm{~K}$ \\
\hline \multicolumn{3}{|l|}{ L-alanine } & \multicolumn{3}{|l|}{ DL-alanine } \\
\hline \multirow{2}{*}{$x=0.33(3)$} & 708 & 670 & $x=0.33(3)$ & 510 & 586 \\
\hline & 471 & 486 & $0.16(6)$ & 656 & 609 \\
\hline $0.09(09)$ & 627 & 564 & $0.09(09)$ & 375 & 428 \\
\hline \multicolumn{3}{|l|}{ L-glutamic acid } & \multicolumn{3}{|c|}{ DL-glutamic acid } \\
\hline$x=0.33(3)$ & 333 & 345 & \multirow{2}{*}{$\begin{aligned} x= & 0.33(3) \\
& 0.20\end{aligned}$} & 378 & 387 \\
\hline 0.20 & 356 & 379 & & 399 & 401 \\
\hline $0.16(6)$ & 383 & 394 & $0.16(6)$ & 418 & 407 \\
\hline $0.11(1)$ & 446 & 453 & $0.11(1)$ & 420 & 412 \\
\hline $0.09(09)$ & 470 & 484 & $0.09(09)$ & 441 & 423 \\
\hline
\end{tabular}

\section{Theoretical model}

The theoretical basis of stereoselectivity of energy transfer between rare earth ions was developed by Sztucki and Stręk [3]. Within this model the rate of resonant energy transfer between two ions $A$ and $B$ is given by the expression

$$
P_{A B}=(2 \pi / \hbar)\left|F_{A B}\right|^{2} \int g_{a a^{\prime}}(E) g_{b b^{\prime}}(E) \mathrm{d} E,
$$

where $F_{A B}$ is the amplitude of energy transfer linked with the electronic part of eigenfunctions and $g(E)$ are the normalized line shape functions of ions $A$ and $B$.

The amplitude of energy transfer is given by

$$
F_{A B}=\left\langle i\left|H_{\text {int }}\right| t\right\rangle+\sum_{j}\left\langle i\left|H_{\text {int }}\right| j\right\rangle\left\langle\left[j\left|H_{\text {int }}\right| t\right\rangle \Delta E^{-1}+\ldots=\left\langle i\left|H_{\text {eff }}^{\mathbf{E T}}\right| t\right\rangle,\right.
$$

where $H_{\text {int }}=H_{A B}+H_{A L}+H_{B L}$ is a sum of the Coulomb interaction between ions $A$ and $B$, and the ligand $L$, given by the expansion with respect to the multipole operators $D^{(k)}$. The zeroth order states are given by the product 
$\left|a_{\alpha} b_{\beta} l_{\lambda}\right\rangle=\left|a_{\alpha}\right\rangle\left|b_{\beta}\right\rangle\left|l_{\lambda}\right\rangle$. If the excitation energy is transferred from the ion $A$ to the ion $B$, the initial state takes the form $|i\rangle=\left|a^{*}\right\rangle\left|b_{0}\right\rangle\left|l_{0}\right\rangle$ and the terminal state $|t\rangle=\left|a_{0}\right\rangle\left|b^{*}\right\rangle\left|l_{0}\right\rangle$.

Let the energy transfer rates in crystals with resolved ligands be denoted as $P_{A B}^{L}$ and $P_{A B}^{R}$ for left (L) and right (R) enantiomers, respectively, therefore the stereoselectivity of energy transfer may be defined by

$$
\Delta P_{A B}=P_{A B}^{\mathrm{L}}-P_{A B} .
$$

If we replace $P$ by the energy transfer amplitude $F$ according to definition (2), Eq. (3) can be transformed to

$$
\begin{gathered}
\Delta P_{A B}=P_{A B}^{L}-P_{A B}=\left[\left|f_{A B}^{-}\right|^{2}+\left|h_{A B}^{+}\right|^{2}+2 \operatorname{Re}\left(f_{A B}^{+} \cdot \overline{f_{A B}^{-}}\right)\right. \\
\left.\quad+2 \operatorname{Re}\left(f_{A B}^{+} \cdot \overline{h_{A B}^{+}}\right)\right](2 \pi / \hbar) \int g_{a a^{\prime}}(E) g_{b b^{\prime}}(E) \mathrm{d} E
\end{gathered}
$$

where $f_{A B}$ is the energy transfer (ET) amplitude in a system with resolved ligands

$$
f_{A B}=\left\langle a^{*}\left|\left\langle b_{0}\left|\left\langle l_{0}^{\mathrm{L}}\left|H_{\mathrm{eff}}^{\mathrm{ET}}\right| l_{0}^{\mathrm{L}}\right\rangle\right| b^{*}\right\rangle\right| a_{0}\right\rangle
$$

while $h_{A B}$ is the modified energy transfer amplitude describing ET accompanied by change of the chirality of the ligand taking part in the process

$$
h_{A B}=\left\langle a^{*}\left|\left\langle b_{0}\left|\left\langle l_{0}^{\mathrm{L}}\left|H_{\mathrm{eff}}^{\mathrm{ET}}\right| l_{0}^{\mathrm{R}}\right\rangle\right| b^{*}\right\rangle\right| a_{0}\right\rangle .
$$

Amplitudes $f$ and $h$ have been divided into two parts, one of them independent on the change of ligand chirality and another part changing sign when we go from the left to the right enantiomer. These parts have been denoted by superscripts + and - , respectively.

It is important to note that the amplitude $h_{A B}$ has been neglected in the former paper [3], but within closer analysis it appeared that it can be responsible for some new phenomena which will be discussed in detail in a forthcoming paper.

Let us notice that expression (4) can be considerably simplified for those systems in which $P^{\mathrm{L}}=P^{\mathrm{R}}$. In this case the term proportional to $2 \operatorname{Re}\left(f_{A B}^{+} \cdot \overline{f_{A B}^{-}}\right)$ vanishes as it is equal to $P^{\mathrm{L}}-P^{\mathrm{R}}$. Since the terms $\left|f_{A B}^{-}\right|^{2}$ and $\left|h_{A B}^{+}\right|^{2}$ are positive, the negative value of $\Delta P_{A B}$ may be expected only when $2 \operatorname{Re}\left(f_{A B}^{+} \cdot \overline{h_{A B}^{+}}\right)$is negative and strong enough. When $h_{A B}$ is small, we obtain positive $\triangle P_{A B}$. In that case $P_{A B}^{\mathrm{L}}>P_{A B}$, therefore the ET in resolved systems is more effective than in racemic ones.

\section{Discussion}

The results of decay time measurements of ${ }^{5} \mathrm{D}_{4}$ fluorescence of $\mathrm{Tb}(\mathrm{III})$ ions as a function of $\mathrm{Eu}^{3+}$ concentration in crystals of alanine and glutamic acid clearly indicate that for all samples the decay times were dependent on handedness of complexed ligands. It has to be noted that the decay times for L-handed alanine crystals were longer than for racemic (DL) form for low and high $\mathrm{Eu}$ (III) concentrations but for intermediate concentration this effect was reverse. It was similar at both measured temperatures. A different behaviour was observed for glutamic acid crystals. The $\mathrm{L}$ form decay times for higher concentration of Eu(III) 
ions $(x=0.33,0.20)$ were shorter than for DL form. For lower concentration $(x=0.11,0.09)$ this effect was reverse. It could be interesting to note that temperature dependence of fluorescence lifetimes of $\mathrm{Tb}(\mathrm{III})$ was also dependent of chirality of crystals. For instance for L-alanine $(x=0.33,0.09)$ the lifetimes measured at $300 \mathrm{~K}$ were longer than at $77 \mathrm{~K}$ whereas for $\mathrm{DL}$ form the lifetimes were shorter. An opposite behaviour was observed for L-glutamic acid crystals where the lifetimes measured at $300 \mathrm{~K}$ were shorter than at $77 \mathrm{~K}$. For DL form of glutamic acid the lifetimes measured at $300 \mathrm{~K}$ were shorter than at $77 \mathrm{~K}$ but only for higher concentration of $\mathrm{Eu}(\mathrm{III})$ ions $(x=0.33,0.20)$. For lower concentrations $(x=0.11,0.09)$ this dependence was reverse.

According to the theory of chirality effect on energy transfer between lanthanide ions [3] the process should be more efficient in resolved systems than in racemic ones, $P_{A B}>0$. To get insight into the possible sources of opposite behaviour $\left(P_{A B}<0\right)$ within the framework of inherent chirality of system we have extended this theory. The other possible sources of negative values of $P_{A B}$ may be connected with the structure of crystal and the mechanisms of energy transfer processes occurring between $\mathrm{Tb}(\mathrm{III})$ and $\mathrm{Eu}(\mathrm{III})$ ions. The deactivation of ${ }^{5} D_{4}$ state of $\mathrm{Tb}$ (III) ion is associated not only with $\mathrm{Tb}(\mathrm{III})-\mathrm{Eu}(\mathrm{III})$ energy transfer but also with the $\mathrm{Tb}(\mathrm{III})-\mathrm{Tb}(\mathrm{III})$ energy transfer and the migration of excitation energy between $\mathrm{Tb}(\mathrm{III})$ ions. All these processes may be nonresonant [2] and then characterized by a different temperature dependence. The mechanism of energy transfer depends also on the crystal structure. According to X-ray analysis recently performed by us $[4,5]$ for alanine and glutamic acid crystals both the systems differ significantly. The crystals of lanthanide ions with glutamic acid form polymeric structure in which two different centrosymmetric (for DL) or two noncentrosymmetric (for $\mathrm{L}$ ) subunits are linked by amino acid chains.

The crystals with alanine are characterized by a quite different structure. For resolved form $L$ we have noncentrosymmetric dimer [5] whereas for racemic form DL there are created the centrosymmetric dimers. It must result in different energy transfer processes. Their nature needs further elaborate studies.

\section{References}

[1] H.G. Brittain, Inorg. Chem. 18, 1749 (1979).

[2] J. Legendziewicz, W. Stręk, E. Huskowska, T. Kim Anh, C. Szafrański, in: Excited States of Transition Elements, Ed. by W. Stręk, J. Legendziewicz, B. Jeżowska-Trzebiatowska, World Sci. Publ., Singapore 1989 p. 258.

[3] J. Sztucki, W. Stręk, J. Chem. Phys. 92, 4256 (1990).

[4] C.N. Dao, T. Glowiak, E. Huskowska, J. Legendziewicz, J. Less-Common Met. 136, 339 (1988).

[5] T. Głowiak, J. Legendziewicz, E. Huskowska, to be published. 